, ,

\title{
Multifractal Properties of Return Time Statistics
}

\author{
Nicolai Hadyn \\ Dept. of Mathematics, University of Southern California, Los Angeles
}

José Luevano

Centre de Physique Theorique, Univ. de Aix Marseille II, and Universidad Autonoma Metropolitana, Azcapotzalco, Mexico

Giorgio Mantica

International Center for the Study of Dynamical Systems, Università dell'Insubria, Via Valleggio 11, Como, Italy, and I.N.F.M., unità di Milano, I.N.F.N. sez. Milano, giorgio.mantica@uninsubria.it

Sandro Vaienti

Centre de Physique Théorique, Luminy, Marseille and PHYMAT, Université de Toulon et du Var, France, and Fédération de Recherche des Unités de Mathématiques de Marseille

(November 1, 2018)

\begin{abstract}
Fluctuations in the return time statistics of a dynamical system can be described by a new spectrum of dimensions. Comparison with the usual multifractal analysis of measures is presented, and difference between the two corresponding sets of dimensions is established. Theoretical analysis and numerical examples of dynamical systems in the class of Iterated Functions are presented.
\end{abstract}

PACS numbers: 05.45.-a 05.45.Df 05.45.Tp

Suppose that $T$ is a transformation of the space $X$ into itself, which preserves the probability measure $\mu$. Let $A$ be a subset of $X$, and $x$ a point in $A$. We let $\tau_{A}(x)$ be the (integer) time of the first return of $x$ in $A$ :

$$
\tau_{A}(x)=\inf \left\{n>0 \text { s.t. } T^{n}(x) \in A\right\} .
$$

Poincare theorem guarantees that the return time is almost certainly finite with respect to any invariant measure. It can be rather long, and particularly short as well: if $x$ is a fixed point of $T$, then obviously $\tau_{A}(x)=1$ for any set $A$ containing $x$. In this paper, we shall prove that the distribution of return times is characterized by multi-fractal properties which can be properly described by the tools of the so-called thermodynamical formalism [1].

Much recent research has been focused on the local statistics of these returns, in the following sense: Take for $A$ a ball of variable radius $\varepsilon$ centered at the point $x$ : $A=B_{\varepsilon}(x)$. Next, consider the cumulative distribution of the first return times, in the ball, of all points of this latter: Let $\lambda(\varepsilon)=\mu\left(B_{\varepsilon}(x)\right)$, and define $m(x, \varepsilon, s):=$ $\mu\left\{y \in B_{\varepsilon}(x)\right.$ s.t. $\left.\lambda(\varepsilon) \tau_{B_{\varepsilon}(x)}(y)>s\right\} / \lambda(\varepsilon)$. In many instances, these statistics have an exponential character: $m(x, \varepsilon, s) \rightarrow e^{-s}$, as $\varepsilon$ tends to zero. Rigorous proofs of this fact have been produced under an ever lessening set of hypotheses [2].

More fundamental for our analysis than the previous, is Kac theorem [3], a classical result of the local analysis: it predicts that, whenever the measure $\mu$ is ergodic with respect to $T$, for any measurable set $A$ (and not just balls), the expectation of $\tau_{A}$ over the set $A$ is just the inverse of the measure of $A$ :

$$
\int_{A} \tau_{A}(y) \frac{d \mu(y)}{\mu(A)}=\frac{1}{\mu(A)}
$$

Furthermore, one must quote the theorem of Ornstein and Weiss [4]: Let $\mathcal{A}$ be a generating partition of $X$, and let us refine it around all the points $x \in X$ by defining the cylinder of order $n, A_{n}(x)$, as the intersection of all the elements of $\mathcal{A}, T^{-1} \mathcal{A}, \ldots, T^{-n+1} \mathcal{A}$ containing $x$. The Ornstein-Weiss theorem states that, whenever the measure $\mu$ is ergodic, the following limit exists $\mu$-almost everywhere, and is equal to $h(\mu)$, the metric entropy of $\mu$ :

$$
\lim _{n \rightarrow \infty} \frac{\log \tau_{A_{n}(x)}(x)}{n}=h(\mu)
$$

This remarkable result is historically the first link between a thermodynamic quantity, and the return times. Parallel to the Ornstein-Weiss theorem, the ShannonMc Millan-Breiman theorem gives the metric entropy by means of the exponential decay of the measure of cylinders around almost all points. It is common in the physical usage to replace cylinders with balls: this allowed in force of the Brin-Katok theorem [5]. The advantage of balls over cylinders, when considering practical applications, is apparent.

Let us therefore replace $A_{n}(x)$ and $n$ in eq. (3) by $B_{\varepsilon}(x)$ and $-\log \varepsilon$, respectively. In the case of Gibbs measures of Axiom-A diffeomorphisms [6], and of a wide class of maps of the interval [7] one has been able to prove rigorously that the modified limit (3) exists $\mu$-almost everywhere, and is equal to the information dimension $D_{\mu}(1)$, of $\mu$ :

$$
\lim _{\varepsilon \rightarrow 0}-\frac{\log \left(\tau_{B_{\varepsilon}(x)}(x)\right)}{\log \varepsilon}=D_{\mu}(1) .
$$

The definition of $D_{\mu}(1)$, and an informal proof of eq. (4), will be given momentarily. 
So far, the analysis has been local. Quite different-and more complex-is the case of the global statistics that we consider in this paper. We let balls of fixed radius $\varepsilon$ cover $X$, and we compute the integrals

$$
\Gamma_{\tau}(\varepsilon, q):=\int_{X} \tau_{B_{\varepsilon}(x)}^{1-q}(x) d \mu(x)
$$

where $q$ is a real quantity. These are the statistical moments of the time required to come back to a neighborhood of the starting point: it is clear that local analysis alone has little to say about the scaling, in the radius $\varepsilon$, of these quantities.

Indeed, $\Gamma_{\tau}(\varepsilon, q)$ are a sort of partition function, quite akin to those employed in the thermodynamical formalism. Their scaling for small $\varepsilon$ defines a set of dimensions, $D_{\tau}(q)$ :

$$
\Gamma_{\tau}(\varepsilon, q) \sim \varepsilon^{D_{\tau}(q)(q-1)},
$$

that we call return time dimensions. The study of these dimensions is the object of this Letter. In level of importance, the first question is about their relations with the usual quantities of the thermodynamical formalism.

Let us first observe that the return time $\tau_{B_{\varepsilon}(x)}(x)$ can be interpreted as a "single-point" sample of the integral in Kac theorem, eq. (2). Therefore, one could estimate that

$$
\tau_{B_{\varepsilon}(x)}(x) \sim \mu\left(B_{\varepsilon}(x)\right)^{-1} .
$$

It is worth recalling now that the local dimension $\alpha(x)$ of the measure $\mu$ at the point $x$ is defined by the scaling $\mu\left(B_{\varepsilon}(x)\right) \sim \varepsilon^{\alpha(x)}$, in the limit of small $\varepsilon$. Moreover, the relation $\alpha(x)=D_{\mu}(1)$ holds almost everywhere in $X$, as discussed above. This fact, and eq. (7), then imply the theorem expressed by eq. (4).

The approximate equality (7) has been already adopted in [8,9] to evaluate via eq. (5), the exact thermodynamical partition function $\Gamma_{\mu}(\varepsilon, q)$,

$$
\Gamma_{\mu}(\varepsilon, q):=\int_{X} \mu\left(B_{\varepsilon}(x)\right)^{q-1} d \mu(x) \sim \varepsilon^{D_{\mu}(q)(q-1)},
$$

whose scaling for small $\varepsilon$ gives the well-known spectrum of generalized dimensions $D_{\mu}(q)$ [10].

The substitution of eqs. (8) by eqs. (5, 6) is particularly significant, for it allows one to treat dynamical systems endowed with physical measures of the SinaiBowen-Ruelle type: in this case the integral can be replaced by a Birkhoff sum over the trajectory $x_{l}:=T^{l}\left(x_{0}\right)$, $l=0, \ldots$, of a generic point $x_{0}$ :

$$
\Gamma_{\tau}(\varepsilon, q)=\lim _{n \rightarrow \infty} \frac{1}{n} \sum_{j=0}^{n-1} \tau_{B_{\varepsilon}\left(x_{j}\right)}^{1-q}\left(x_{j}\right)
$$

Indeed, this Birkhoff procedure, eq. (9), is the one originally employed in [8.9], where it is claimed to produce the spectrum of measure dimensions $D_{\mu}(q)$.
It must be remarked that eq. (9) considers a single sum along the orbit, while in the usual Grassberger-Procaccia technique for the $q$-correlation integrals, one must work with a double summation, where all the couples of points along the same (or different) orbit are compared [11].

We are now ready to introduce the main result of this paper: contrary to the usage of $[8,9$, the estimate (7) does hold only for the first moment, as in Kac theorem, but not in the stronger sense required in eqs. (5), and (8): the variables $\tau_{B_{\varepsilon}(x)}$ and $\mu\left(B_{\varepsilon}(x)\right)^{-1}$ have different large deviation properties. Therefore, $D_{\mu}(q)$ and $D_{\tau}(q)$ are not the same function, and the latter defines a new bona fide spectrum of dimensions.

The abstract proof of this statement would be little informative, if not paralleled by a specific example. It is therefore convenient to introduce a family of dynamical systems whose invariant measures are completely known, in the sense that the spectrum of dimensions $D_{\mu}(q)$ can be easily and precisely computed: these are the so-called systems of iterated functions, or I.F.S., see [12 for details. In the simplest, one-dimensional case, an I.F.S. consists of a collection of contracting maps of the line, $\phi_{i}: \mathbf{R} \rightarrow \mathbf{R}, i=1, \ldots, M$, where $M$ is the number of maps. These latter can be thought of as the inverse branches of the dynamics: $\left(T \circ \phi_{i}\right)(x)=x$ for any $i$. In so doing, $T$ is characterized by a mixing repeller, that is also the attractor of the collective action (in the sense that will be made soon clear) of the maps in the I.F.S..

A family of invariant measures for $T$ can be constructed assigning a probability, $\pi_{i}$, to each map $\phi_{i}$, $\sum_{i=1}^{M} \pi_{i}=1$. The dynamics of I.F.S. can be constructed by sequentially applying maps $\phi_{\sigma}$, where $\sigma$ is chosen in a random fashion with probability $\pi_{\sigma}$. Any orbit of the probabilistic I.F.S., when time-reversed, becomes an orbit of the deterministic dynamics $T$. Notice that return times are invariant under time reversal.

The reader will find useful to consider the usual ternary Cantor set measure as the invariant measure of the following 2-maps I.F.S.: $\phi_{1}(x)=x / 3, \phi_{2}(x)=(x+2) / 3$, $\pi_{1}=\pi_{2}=1 / 2$. The related invariant measure is an example of mono-fractal: all generalized dimensions are equal to the constant $\delta=\frac{\log 2}{\log 3}$. Let us perform the return time analysis of this dynamical system following the Birkhoff sums approach, eq. (9). Other techniques of estimating the integral (5) will be presented elsewhere.

We can first verify that the scaling relation (6) holds: fig. 1 assures us that this is indeed the case.

We can then extract the return dimensions $D_{\tau}(q)$ versus $q$ : the numerical results are plotted in fig. 2. They reveal a non-trivial range of dimensions: as far as return times are concerned, the dynamics is multifractal. Moreover, the return time dimensions are consistent with the constant value $\delta$ (within the statistical error bars) for negative dimensions, but are significantly different from this latter for positive values of $q$, the more, the larger the value of $q$.

Resorting now to exact analysis, we can confirm these 
results: precisely, we are able to prove that i) $D_{\mu}(0)=$ $D_{\tau}(0)$, and ii) $D_{\tau}(q) \rightarrow 0$ for large $q$. The second fact proves that the return dimensions $D_{\tau}(q)$ are significantly different from measure dimensions, while the first hints at relations that, at least in certain dynamical systems, may exist between them.

To see that $i$ ) is true, observe that the repeller of $T$ can be hierarchically covered by intervals $A_{l}^{k}$, of length $1 / 3^{k}$, where $k=0, \ldots$ is the index of the generation, and $l=1, \ldots, 2^{k}$ is the label of the single interval within its generation. This is simply the usual construction of the ternary Cantor set, in which one carves a hole of length $1 / 3^{k}$ in the middle of each interval generation $k-1$.

For a fixed value of $k$, let us choose $\varepsilon=\varepsilon_{k}=1 / 3^{k}$. Then, simple geometric considerations assure us that whenever $x$ belongs to $A_{l}^{k}$, with $k$ and $l$ fixed, it is also contained in the ball of radius $\varepsilon_{k}$ centered around any other point in $A_{l}^{k}$. In addition, these balls intersect no interval of generation $k$ other than $A_{l}^{k}$. This permits us to employ Kac theorem, eq. (2), to compute exactly the sum $\Gamma_{\tau}\left(\varepsilon_{k}, 0\right)$ :

$$
\Gamma_{\tau}\left(\varepsilon_{k}, 0\right)=\sum_{l=0}^{2^{k}} \int_{A_{l}^{k}} \tau_{A_{l}^{k}}(x) d \mu(x)=2^{k} .
$$

It is then clear that $i$ ) holds.

Remark that the intervals $A_{l}^{k}$, are also the cylinders of order $k$ of the dynamics. Therefore, the result we have just proven also applies to the return time dimensions, when defined via cylinders, and predicts that the dimension of order zero is equal to the topological entropy. Following the same idea, one also expects that the cylinder return time dimensions will be related to the Renyi entropies of the measure [13].

Observe that the above results are by no means restricted to the one-dimensional case. At the same time it is evident that use of Kac theorem is permitted only for $q=0$, for otherwise different fluctuation properties of the return time statistics set in. This leads us directly to the statement $i$ i).

Remark that the return times define partitions of $X$ by the sets $R_{n}(\varepsilon):=\left\{x \in X\right.$ s.t. $\left.\tau_{B_{\varepsilon}(x)}(x)=n\right\}$. This allows us to re-write the moments $\Gamma_{\tau}(\varepsilon, q)$ as a new summation:

$$
\Gamma_{\tau}(\varepsilon, q)=\sum_{n=1}^{\infty} n^{1-q} \mu\left(R_{n}(\varepsilon)\right)
$$

When $q$ is large, terms with small $n$ lead the sum (11). Let us retain just the first of these: it does not vanish by increasing $q$. This term is the measure of $R_{1}(\varepsilon)$, the set of points that move less than $\varepsilon$ in a single iteration. Clearly, all fixed points of $T$ belong to this set. Let $\bar{x}$ be any one of these, and let us assume that $\varepsilon$ is small enough, and that the transformation $T$ is smooth. Then, $R_{1}(\varepsilon)$ contains the ball of radius $\varepsilon / \Lambda$ centered at $\bar{x}$, where $\Lambda$ is the largest singular value of the matrix $\mathbf{1}-T^{\prime}, \mathbf{1}$ is the identity, and $T^{\prime}$ is the Jacobian matrix of $T$ at $\bar{x}$.
Moreover, the ball of radius $\varepsilon / \sigma$, where $\sigma$ is now the smallest singular value, contains the connected part of $R_{1}(\varepsilon)$ around $\bar{x}$. Then, we can estimate that, for large $q$,

$$
\Gamma_{\tau}(\varepsilon, q) \simeq \sum_{\bar{x} \text { s.t. } T(\bar{x})=\bar{x}} \mu\left(B_{\rho \varepsilon}(\bar{x})\right),
$$

where the sum is extended to all fixed points of $T$, and where $\rho$ is a multiplicative factor, which depends on $\bar{x}$ : $\sigma<\rho<\Lambda$. When the number of terms in the sum is finite, the leading contribution to (12) comes from the fixed point $\bar{x}$ with the smallest local dimension, $\alpha_{m}$ : since $\mu\left(B_{\varepsilon}(\bar{x})\right) \sim \varepsilon^{\alpha_{m}}$, it follows at once that

$$
D_{\tau}(q) \simeq \frac{\alpha_{m}}{q-1}, \text { when } q \rightarrow \infty .
$$

We can readily verify that eq. (13) is satisfied by the Cantor set dynamics introduced above. The fixed points of $T$ are here zero, and one, and they are both characterized by the same value of the local dimension, $\alpha_{m}=\frac{\log 2}{\log 3}$. In fig. 2 the relation (13) is validated by the numerical results of $D_{\tau}(q)$ for large $q$.

This investigation is not limited to a single dimension. We now add a two-dimensional example with a non-trivial dimension function $D_{\mu}(q)$ : the motion on a Sierpinsky gasket, corresponding to the I.F.S. maps $\phi_{1}(x, y)=(x / 2, y / 2), \phi_{2}(x, y)=((x+1) / 2,(y+1) / 2)$, and $\phi_{3}(x, y)=(x / 2,(y+1) / 2)$, with non-uniform probabilities $\pi_{1}=4 / 10, \pi_{2}=\pi_{3}=3 / 10$. In fig. 3 we have compared the exact thermodynamical function $D_{\mu}(q)$ and the numerically evaluated $D_{\tau}(q)$. For negative values of $q$ the two dimensions are very close, and discrepancies may be due to the finite length of the Birkhoff sum, and, more importantly, of the fitting interval in $\varepsilon$, which cannot be accounted for by the statistical definition of the error bars. For positive values of $q$, to the contrary, the two dimensions are rather different, and the asymptotic formula (13) soon becomes a very close approximation of $D_{\tau}(q)$.

In conclusion, we have shown that the statistics of return times are characterized by multi-fractal properties, well defined by a new spectrum of dimensions $D_{\tau}(q)$. We have also found that, contrary to implicit previous statements in the literature, this spectrum does not coincide with the usual multi-fractal spectrum $D_{\mu}(q)$. It is now interesting to understand the meaning of the Legendre transforms associated to these new dimensions. We have proven that $D_{\tau}(0)=D_{\mu}(0)$ under certain hypotheses on the dynamical system. Approximate equality seems to hold for negative $q$ 's as well 16. This might provide a means for computing negative measure dimensions, which is known to be a challenging numerical task. Finally, we have shown that in a large class of systems the relation $D_{\tau}(q) \simeq \frac{\alpha_{m}}{q-1}$ holds for large $q$, where $\alpha_{m}$ is the smallest local dimension at the fixed points of $T$. 
[1] Y. G. Sinai, Usp. Mat. Nauk. 27, 21 (1972); R. Bowen, Lect. Notes Math 470, 1 (1975); D. Ruelle, Statistical Mechanics, Thermodynamic Formalism (AddisonWesley, Reading, MA, 1978).

[2] M. Hirata, B. Saussol, S. Vaienti, Comm. Math. Phys. 206 (1999) 33-55; A. Galves, B. Schmitt, Random Comput. Dyn. 5 (1997) 337-348; N. Hadyn, S. Vaienti, The limiting distribution and error terms for return times of dynamical systems (submitted, 2001).

[3] M. Kac, "Probability and related topics in physical sciences" (1959) AMS

[4] D. Ornstein, B. Weiss, IEEE Trans. inf. Theory, 39 (1993) 78-83

[5] M. Brin, A. Katok, "On local entropy", Geometric dynamics (Rio de Janeiro, 1981), 30-38, Lecture Notes in Math., 1007, Springer, Berlin-New York, 1983

[6] L. Barreira, B. Saussol, Comm. Math. Phys 219 (2001) 443-464.

[7] B. Saussol, S. Troubetzkoy, S. Vaienti, Recurrence, Dimension, and Lyapunov Exponent, J. Stat. Phys., to appear.

[8] P. Grassberger, Phys. Lett. A, 97 (1983) 227-230.

[9] M.H. Jensen, L.P. Kadanoff, A. Libchaber, I. Procaccia, and J. Stavans, Phys. Rev. Lett. 55 (1985) 2798-2801.

[10] Ya. Pesin, "Dimension theory in dynamical systems", The University of Chicago Press, 1997.

[11] P. Grassberger, I. Procaccia, Phys. Rev. Lett., 84 (1983) 346-349

[12] M.F. Barnsley and S.G. Demko, Proc. R. Soc. London A 399 (1985) 243-275.

[13] If one considers cylinders in place of balls, the two generalized dimensions can be identified with the deviation functions of the variables $\tau_{A_{n}(x)}(x)$ and $\mu\left(A_{n}(x)(x)\right)$ [14. Then, one can rigorously prove that the two dimensions coincide in a small neighbourhood to the right of $q=1$, but they must differ for large $q$ 15.

[14] P. Collet, A. Galves, and B. Schmitt, Nonlinearity 12 (1999) 1225-1237.

[15] N. Hadyn and S. Vaienti, preprint (2001).

[16] A precise numerical investigation of this point requires more sophisticated integration techniques than Birkhoff sums: see e.g. G. Mantica, Constr. App. 12, (1996) 509530 .

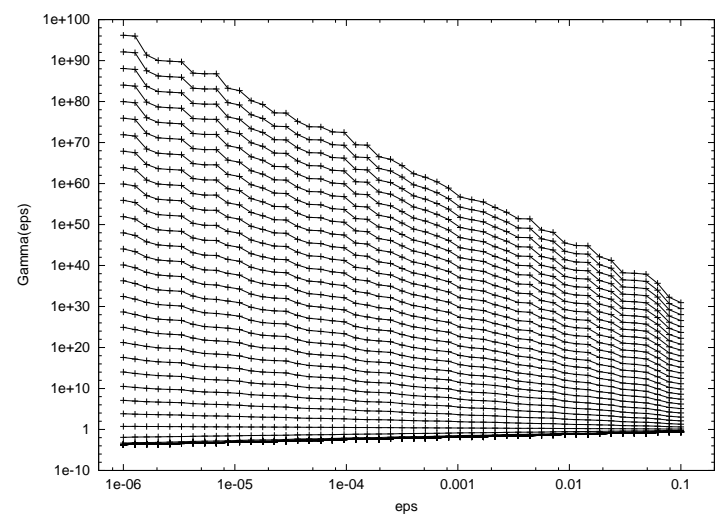

FIG. 1. Return time partition sums $\Gamma_{\tau}(\varepsilon, q)$ versus $\varepsilon$ for a set of equally spaced values of $q$ ranging from $q=-19$ (bottom curve) to $q=19$ (top). Lines in between points are solely intended to connect data with the same value of $q$.

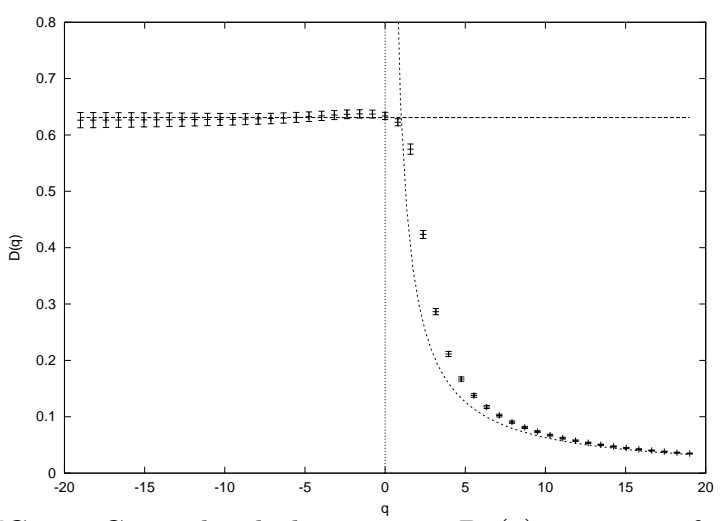

FIG. 2. Generalized dimensions $D_{\tau}(q)$ versus $q$ for the ternary Cantor set dynamics. Error bars are defined via the uncertainty in the least square fits of Fig. (11), under the usual statistical assumptions. The horizontal line is the (flat) measure thermodynamics : $D_{\mu}(q)=\log 2 / \log 3$. The dashed curve for $q>0$ is eq. (13) with $\alpha=\log 2 / \log 3$.

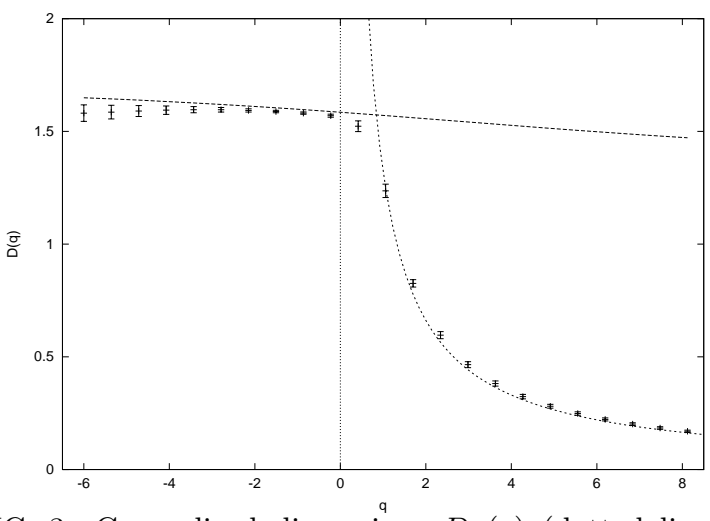

FIG. 3. Generalized dimensions $D_{\mu}(q)$ (dotted line) and $D_{\tau}(q)$ (error bars) versus $q$ for the Sierpinsky dynamics described in the text. Data have been computed by least square fits of Birkhoff sums with $n=800000$. Also reported for positive $q$ is the asymptotic formula (13). 